Review

\title{
Stat3 and Gap Junctions in Normal and Lung Cancer Cells
}

\section{Stephanie Guy ${ }^{1, \dagger}$, Mulu Geletu ${ }^{2, \dagger, *}$, Rozanne Arulanandam ${ }^{1, \S}$ and Leda Raptis ${ }^{1,2, *}$}

1 Department of Pathology, Queen's University, Kingston, ON K7L 3N6, Canada;

E-Mail: 11sg27@queensu.ca

2 Department of Biomedical and Molecular Sciences, Queen's University, Kingston, ON K7L 3N6, Canada

$\dagger$ These authors contributed equally to this work.

$\$$ Present address: Department of Chemical and Physical Sciences, University of Toronto, Mississauga, William Davis 3126B Room 3023, 3359 Mississauga Rd. N, Mississauga, ON L5L 1C6, Canada; E-Mail: mulu.geletu@utoronto.ca.

$\S$ Present address: Department of Pathology and Molecular Medicine, Centre for Innovative Cancer Therapeutics, Ottawa Hospital Research Institute, 501 Smyth Road, Ottawa, ON K1H 8L6, Canada; E-Mail: pinklemonade79@gmail.com.

* Author to whom correspondence should be addressed; E-Mail: raptisl@queensu.ca; Tel.: +1-613-533-2462.

Received: 14 November 2013; in revised form: 11 February 2014 / Accepted: 27 February 2014 / Published: 25 March 2014

\footnotetext{
Abstract: Gap junctions are channels linking the interiors of neighboring cells. A reduction in gap junctional intercellular communication (GJIC) correlates with high cell proliferation, while oncogene products such as Src suppress GJIC, through the Ras/Raf/Erk and other effector pathways. High Src activity was found to correlate with high levels of the Src effector, Signal Transducer and Activator of Transcription-3 (Stat3) in its tyrosine-705 phosphorylated, i.e., transcriptionally activated form, in the majority of Non-Small Cell Lung Cancer lines examined. However, Stat3 inhibition did not restore GJIC in lines with high Src activity. In the contrary, Stat3 inhibition in normal cells or in lines with low Src activity and high GJIC eliminated gap junctional communication. Therefore, despite the fact that Stat3 is growth promoting and in an activated form acts like an oncogene, it is actually required for junctional permeability.
} 
Keywords: signal transducer and activator of transcription-3; gap junctions; connexin43

\section{Introduction}

Gap junctions are channels linking the interiors of neighboring cells that allow the passage of ions and small metabolites. They consist of integral membrane proteins, the connexin gene family, comprising 21 known members in humans [1]. Connexins play a critical role in the regulation of development, contraction, homeostasis, as well as cell growth and differentiation [2].

Connexins are composed of four transmembrane domains, two extracellular loops with six conserved cysteine residues, a cytoplasmic loop and cytoplasmic amino- and carboxy-termini. The carboxy-terminus varies in length and provides sites for protein interactions [2]. Connexin-43 (Cx43) is a ubiquitous connexin which is present in at least 43 tissues and 46 different cell types, and is the predominant connexin in most cultured cell lines $[3,4]$. Cx43 has an unusually short half-life $(1-3 \mathrm{~h})$, both in cell culture and in tissues, with a high turnover [5]. Like most integral membrane proteins, $\mathrm{Cx} 43$ is cotranslationally inserted into the endoplasmic reticulum during its biosynthesis, where interloop disulphide bonds form. $\mathrm{Cx} 43$ then enters the trans-Golgi network, where it oligomerises into hexamers, called connexons, which migrate to the cell surface where they coalesce at sites nucleated for gap junction assembly [6,7]. Following their formation, gap junctions are internalised by a mechanism where the entire gap junction or fragments thereoff form a double-membrane structure, termed connexosome, which merges with lysosomes where they are degraded $[2,3,6]$.

A number of oncogene products such as v-Src [8], the middle tumor antigen of polyoma virus (mT, which transforms through $\mathrm{cSrc}$ activation [9,10]), the activated chaperone Hsp90N [11], activated Ras [12], v-mos [13] and others were shown to interrupt gap junctional, intercellular communication (GJIC). Some of these oncogenes have kinase activity and phosphorylate $\mathrm{Cx} 43$ on tyrosine or serine/threonine sites. In fact, $\mathrm{Cx} 43$ is highly phosphorylated. Activation of kinases such as protein kinase A (PKA) and protein kinase CK1 increases Cx43 phosphorylation and gap junction transfer, while activation of protein kinase C, p34cdc2 (cyclin B kinase), Erk1/2 and Src decrease GJIC [14-16].

$s r c$ is the first oncogene to be discovered, as the tumor gene of the Rous sarcoma virus. $s r c$ encodes a protein with tyrosine kinase activity $(S r c)$, which correlates with tyr-418 phosphorylation $(S r c 418)$. $S r c$ is often activated in cancer. As such, Src is one of the best studied targets for cancer therapy. Results from a number of labs indicated that the Src kinase can affect Cx43 phosphorylation either directly on tyrosine, or through its effector pathways on serine or threonine. At first the Src-homology-3 (SH3) domain of Src binds a proline-rich sequence of $\mathrm{Cx} 43$, Pro-274 to Pro-280. The proximity between the two proteins facilitates phosphorylation of $\mathrm{Cx} 43$ at tyr-265 by Src. This creates a binding site for the Src, Src-homology-2 (SH2) domain that facilitates phosphorylation of tyr-247 of Cx43, triggering channel closure [8,16]. Still, the role of $S r c$, as opposed to its effectors, upon GJC in cancer is unclear. The $S r c$ effectors, PLC $\gamma$ (which activates protein kinase C, PKC) and Erk1/2 also suppress GJIC in their own right, through phosphorylation at serines 368 and 372, or 255, 279 and 282, respectively. In fact, the Ras/Raf/Erk pathway was shown to transduce Src signals leading to GJIC suppression [17]. 
A prominent Src effector is the Signal Transducer and Activator of Transcription-3 (Stat3) [18], a key cytoplasmic signal transducer which is often overactive in cancer. A constitutively active form of Stat3, Stat3C, can transform cultured mouse fibroblasts, pointing to an etiological role of Stat3 in cancer [19]. Besides the Src family, a number of growth factor receptors such as the EGF family including Her2/ErbB2 and receptors for cytokines such as the IL6 family phosphorylate Stat3 at tyr-705 (Stat3-705). This triggers Stat3 dimerisation through a reciprocal interaction between the SH2 domain and ptyr-705 of Stat3, migration to the nucleus and activation of transcription of specific genes involved in cell division and survival, such as myc, Bcl-xL, Mcl-1, survivin, HGF [20] and others (reviewed in [21]). Recent results from our lab and others demonstrated that engagement of cadherins, cell to cell adhesion molecules, as occurs in cultured cells grown to high densities, can dramatically increase Stat3, tyr-705 phosphorylation and transcriptional activity [22-24]. This was found to be triggered by a dramatic increase in the levels of the Rac1 and Cdc42 GTPases through inhibition of their proteasomal degradation, and this leads to transcriptional upregulation of the interleukin-6 (IL6) gene, triggering Stat3, tyr-705 phosphorylation and activation ([22], reviewed in [25,26]).

Despite the extensive literature on the effect of oncogenes upon GJIC, the effect of Stat3 upon GJIC in cancer cells is unclear. Here, we summarise our recent findings on the effect of the $\mathrm{Src} / \mathrm{Stat} 3$ axis upon GJIC in cells transformed in culture and in lines established from Non-Small Cell Lung Cancer (NSCLC) tumors. Although in the majority of lines high Src418 correlated with high Stat3-705 and the absence of GJIC, Stat3 inhibition did not restore communication in any of the lines examined. This is in sharp contrast to inhibition of the Ras pathway, which did increase GJIC [17]. In the contrary, Stat3 inhibition in lines with extensive GJIC eliminated junctional permeability, indicating that, despite the fact that in an activated form it can act as an oncogene [19], Stat3 is actually required for gap junctional communication.

\section{GJIC Examination}

A reliable method to measure gap junctional permeability is a prerequisite for the investigation of the effect of Stat3 upon GJIC. GJIC examination is usually conducted through the introduction of a fluorescent tracking dye such as Lucifer yellow (LY) by microinjection, scrape-loading [27] or preloading with dye [28], followed by observation of its migration into neighboring cells, or by measuring the recovery of fluorescence after photobleaching [29]. These methods are generally expensive and time-consuming or introduce the potential complication of cellular damage. To overcome these problems, we developed a powerful technique where cells are grown on a glass slide, half of which is coated with electrically conductive, optically transparent, Indium-Tin oxide (ITO) [30]. A LY solution is added to the cells and an electrical pulse delivered. The pulse opens transient pores on the plasma membrane through which LY enters the cell, then rapidly reclose with no detectable damage to the cell. As a result, cells growing on the conductive side of the slide are loaded with LY through electroporation, while cells on the adjoining, non-conductive area do not receive any current, therefore are not permeabilized. The LY can then diffuse through gap junctions into these cells, forming a gradient of fluorescence. Following washing, the cells are observed under phase contrast and fluorescence illumination. Tracer movement can be evaluated several minutes after the electrical pulse, by overlapping the phase contrast and fluorescence images of the cells [9,31,32]. Gap junctional 
communication can be precisely quantitated in this way, simultaneously and in a large number of cells, without any detectable disturbance to cellular metabolism, presumably because the pores reclose rapidly, so that the cellular interior is restored to its original state. The average number of cells into which LY has transferred, per cell loaded with LY by electroporation is the GJIC value. Normally, the transfer from at least 200 cells is calculated [33-35]. The equipment (ACE-100, InSitu Porator apparatus) is available from Cell Projects Ltd. (Harrietsham, Kent, UK).

Electroporation in situ was employed to examine the role of Stat3 upon GJIC, both in immortalised rat liver epithelial T51B [36] or mouse lung epithelial type II E10 [37] cells both of which normally have extensive GJIC, and in lung cancer lines or primary tumor cells expressing different levels of activated $\operatorname{Src}[33,38]$.

\section{Stat3 and GJIC in Cultured, Normal and Src-Transformed Cells}

\subsection{Cell Density Upregulates GJIC and Connexin-43 Protein Levels}

The formation of gap junctions depends upon cell to cell contact, and the engagement of cadherins into adherens junctions [39]. Therefore, it is not surprising that in immortalised rat liver epithelial T51B or mouse lung E10 cells [37], GJIC showed a substantial increase from approximately 1.1 to 6, when cells were grown to densities from $90 \%$ to 3 days post-confluence [33]. Interestingly, the levels of total $\mathrm{Cx} 43$ protein also increased dramatically with density and plateaued at $\sim 2$ days after $100 \%$ confluence. These data indicate that cell to cell contact can trigger a significant increase in GJIC and $\mathrm{Cx} 43$ levels. Therefore, in all subsequent experiments GJIC was examined at 3 days post-confluence [33].

\subsection{Stat3 Does Not Transduce Src Signals to GJIC Suppression}

We at first examined the effect of Stat 3 upon GJIC in in vitro transformed, T51B cells where Src was activated through $\mathrm{mT}$ expression (T51B-Src cells) [36]. Stat3 was downregulated through treatment with the pharmacological inhibitors CPA7 [40,41] or 23I-201 [38,42], or through expression of shRNA with a retroviral vector [36]. Given that Stat3 is an effector of $S r c$, a tyrosine kinase with the ability for GJIC suppression, and an oncogene in its own right [19], it was expected that Stat3 might transduce Src signals leading to GJIC suppression. However, the results demonstrated that Stat3 downregulation does not restore GJIC, indicating that the high Stat3 activity in T51B-Src cells cannot be responsible for their lack of junctional communication. This is in sharp contrast to Ras, whose inhibition restored GJC in Src-transformed rodent fibroblasts, and consistent with findings by Ito et al. [17].

\subsection{Stat3 Is a Positive Regulator of GJIC and Cx43 Levels}

We next investigated whether Stat3 might, in fact, play a positive role upon GJIC, by examining the effect of Stat3 inhibition in the parental T51B cells. Interestingly, Stat3 downregulation essentially eliminated GJIC in T51B cells [36]. Conversely, expression of the constitutively active form of Stat3, Stat3C [19] increased the already extensive gap junctional communication in T51B cells $([33,43])$. Taken together, these data indicate that Stat 3 does in fact play a positive role in the maintenance of gap junction function. This is in sharp contrast to Ras, which was shown to suppress GJIC in non-transformed 
rodent fibroblasts [12]. Therefore, rather than increasing GJIC, Stat3 inhibition eliminates junctional permeability, indicating that Stat 3 activity is actually required for gap junction function.

As noted above, the half-life of $\mathrm{Cx} 43$ is $1-3 \mathrm{~h}$, while cell density (which increases Stat3-705) causes a dramatic increase in $\mathrm{Cx} 43$ levels in non-transformed cells. Interestingly, Stat 3 downregulation through CPA7 treatment or shRNA expression caused a dramatic reduction in Cx43 levels in rat liver epithelial T51B, mouse lung E10 cells or rat F111 fibroblasts at all densities examined, concomitant with GJIC reduction. These findings indicate that Stat 3 activity is required for the maintenance of Cx43 protein levels [33].

\section{Src as a Stat3 Activator in Non-Small Cell Lung Cancer}

\subsection{Cell Density Increases Stat3, in a Src-Independent Manner}

Examination of the levels of tyr-418 phosphorylated, i.e., activated $\operatorname{Src}(\operatorname{Src} 418)$ in a number of NSCLC biopsies revealed the presence of higher Src activity than the surrounding, non-tumor lung tissue $[44,45]$. However, Src's contribution to Stat3 activity in NSCLC lines and primary cells which may express other oncogenes in addition to $S r c$ is a matter of controversy. Examination of Stat3 levels in certain NSCLC lines demonstrated that $S r c$ is a major Stat3 activator [46], while in another report [47] Src inhibition in different NSCLC lines was found to actually increase Stat3-ptyr705. Since cell density causes a dramatic increase in Stat3-705 [25], differences in confluence might account at least in part for these apparent discrepancies.

Despite the fact that $S r c$ is a potent Stat3 activator, $S r c 418$ levels did not increase with density in T51B or E10 cells, or in the NSCLC lines SK-LuCi6 (Figure 1A) and QUDB that have low Src levels, before or after expression of activated Src (lines T51B-Src, E10-Src, SK-LuCi6-Src and QUDB-Src, respectively). Moreover, $S r c$ inhibition with the inhibitors dasatinib (Figure 1A,B) or PD180970, or downregulation through expression of a $\operatorname{SrcDN}$ mutant with an adenovirus vector [24,38], or genetic ablation of Src as well as the related Yes and Fyn genes [23] did not reduce the density-dependent, Stat3 upregulation. Taken together, these findings indicate that the density-mediated, Stat 3 activation is independent of Src. Therefore, to avoid the confounding factor of density upon Stat3-705, the correlation between $\operatorname{Src} 418$ and Stat3-705 was examined at 50\% confluence, in a number of NSCLC lines.

\subsection{Src Is a Major Stat3 Activator in Certain NSCLC Lines}

Besides Src, Stat3 is known to be a prominent effector of other cytokine and membrane tyrosine kinase receptors, which may be present in an activated form in NSCLC cells and upregulate Stat3 activity. Therefore, to examine the effect of $S r c$ specifically upon Stat3 the correlation between $\operatorname{Src} 418$ and Stat3-705 levels was at first assessed in a number of NSCLC lines. Nine lines displayed high Src418 levels (100\% to $25 \%$ of SK-LuCi6-Src), which were found to correlate with their levels of Stat3-705 (Table 1) [33,38] pointing to the possibility that $S r c$ may be a significant contributor to Stat3 activity in these lines. At the same time, two lines with low Src418 levels (QUDB and SK-LuCi6) had low Stat3-705. In sharp contrast however, line LC-T displayed high Stat3-705 despite the fact that Src418 was undetectable. Similarly, SK-MES cells had intermediate Src418 levels (25\% of SK-LuCi6-Src), although Stat3-705 was at 90\%, and FR-E cells with $\operatorname{Src} 418$ of $25 \%$ displayed Stat3-705 of $70 \%$, 
indicating that other, Src-independent factor(s) must also be responsible for the high Stat3-ptyr705 in these lines (Table 1).

Figure 1. Density-induced, Stat3 activation is independent of Src. (A) SK-Luci6 cells were grown to densities of $80 \%$ confluence or 2 days post-confluence as indicated and treated with Dasatinib (lanes 3,4) or not (lanes 1,2). Detergent cell extracts were probed for Src418, Stat3-705, or GAPDH as a loading control. Right panel, lysates were probed for total Src, total Stat3 or GAPDH. Numbers under the lanes refer to densitometry values. Numbers at the left refer to Molecular weight markers; (B) SW-1573 cells were grown to the indicated densities and treated with Dasatinib (lanes 4-6) or not (lanes 1-3). Detergent cell extracts were probed for Src418, Stat3-705, or GAPDH as a loading control. Right panel, lysates were probed for total Src, total Stat3 or GAPDH. Numbers under the lanes refer to densitometry values. Numbers at the left refer to Molecular weight markers.

A
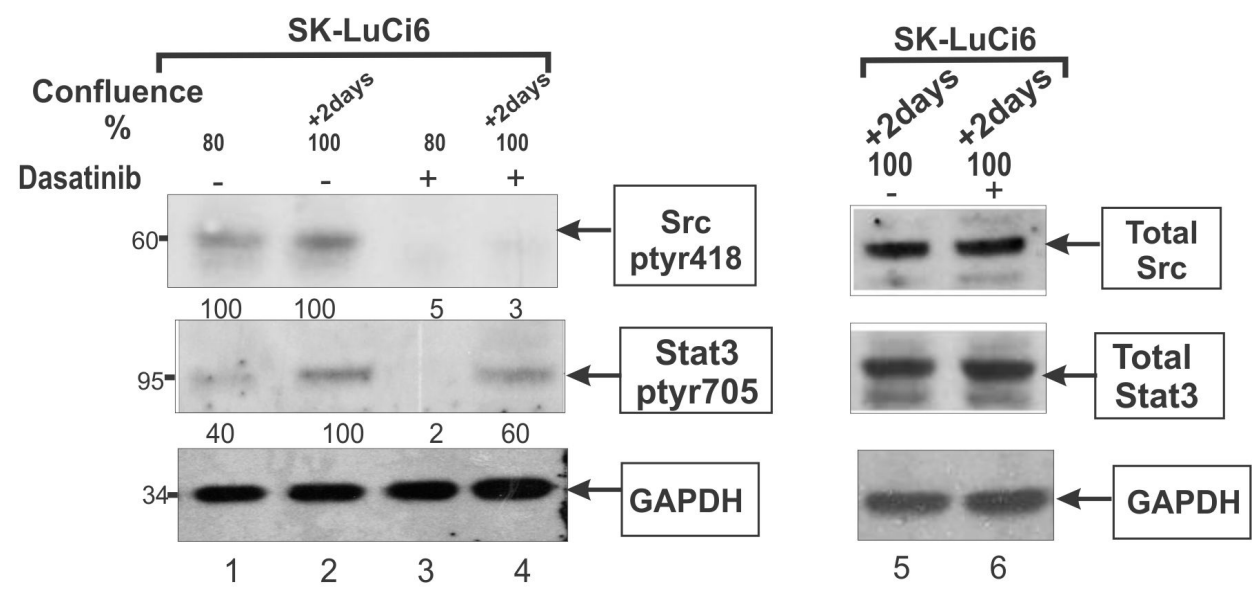

B
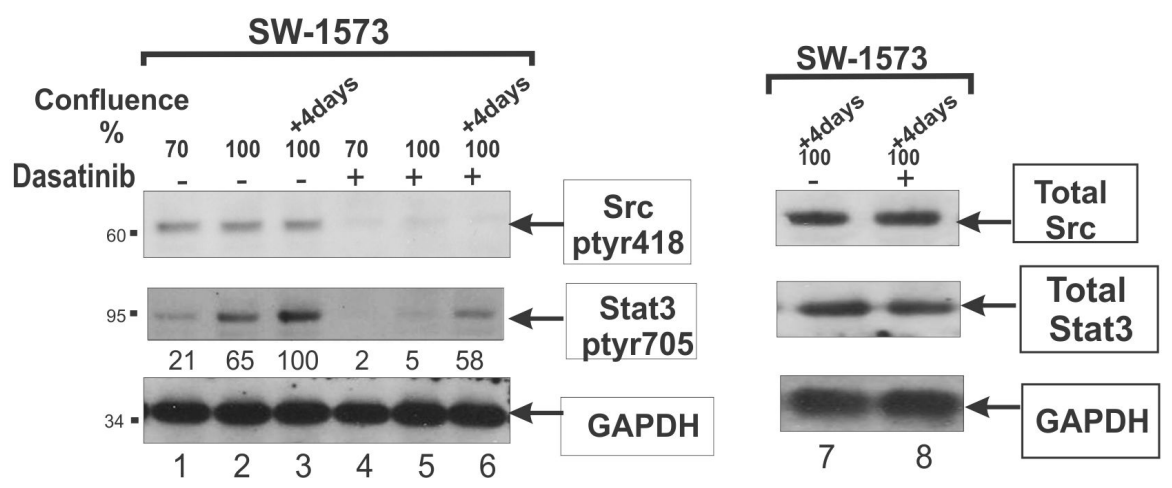

Examination of the actual contribution of Src to Stat3-705 levels was performed by assessing the ability of the Src inhibitors dasatinib or PD180970 to reduce Stat3-705 levels. The results revealed that in sparsely growing cells (e.g., SW-1573) dasatinib caused a dramatic reduction in Stat3-705 (Figure 1B, lane 1 vs. 4 and 2 vs. 5), pointing to $S r c$ as a significant Stat3 activator. However, in confluent cultures the reduction was only $\sim 50 \%$ (lane $3 v s$. 6), which further confirms that the density-induced, Stat 3 activation is independent of Src. Similar results were obtained with 11 lines with high $\operatorname{Src} 418$ 
(Table 1). Consistent with this observation, in cells with low Src418 (e.g., SK-LuCi6 (Figure 1A), QUDB, SHP-77 and BHE), the Stat3-705 increase observed at high confluence was resistant to dasatinib treatment.

Table 1. Src, Stat3 and GJIC in lung cancer lines.

\begin{tabular}{|c|c|c|c|c|}
\hline \multirow{2}{*}{ Cell line } & \multirow{2}{*}{$\operatorname{Src}^{\alpha}(\%)$} & \multicolumn{2}{|c|}{ Stat3 $^{\alpha}(\%)$} & \multirow{2}{*}{ GJIC $^{\beta}$} \\
\hline & & $50 \%$ confluent & $100+3$ days & \\
\hline \multicolumn{5}{|c|}{ Lines with high $S r c-418$ levels } \\
\hline SK-LuCi6-Src & $100 \pm 12$ & $100 \pm 10$ & $420 \pm 33$ & $0.2 \pm 0.1$ \\
\hline A549 & $95 \pm 11$ & $93 \pm 12$ & $320 \pm 32$ & $0.3 \pm 0.1$ \\
\hline SK-Lu1 & $85 \pm 5$ & $90 \pm 11$ & $311 \pm 23$ & $1 \pm 0.2$ \\
\hline Calu-1 & $96 \pm 9$ & $100 \pm 10$ & $290 \pm 12$ & $0.1 \pm 0.1$ \\
\hline SW-900 & $100 \pm 13$ & $100 \pm 12$ & $405 \pm 21$ & $0.1 \pm 0.1$ \\
\hline Calu-6 & $95 \pm 10$ & $90 \pm 12$ & $300 \pm 18$ & $0.1 \pm 0.1$ \\
\hline SW-1573 & $70 \pm 9$ & $70 \pm 8$ & $180 \pm 12$ & $0.2 \pm 0.1$ \\
\hline WT-E & $60 \pm 9$ & $30 \pm 4$ & $80 \pm 11$ & $0.2 \pm 0.1$ \\
\hline BEN & $42 \pm 5$ & $60 \pm 8$ & $182 \pm 25$ & $0.2 \pm 0.1$ \\
\hline H1299 & $30 \pm 4$ & $30 \pm 5$ & $60 \pm 18$ & $0.2 \pm 0.1$ \\
\hline FRE & $25 \pm 3$ & $70 \pm 4$ & $180 \pm 25$ & $0.5 \pm 0.1$ \\
\hline SK-MES & $25 \pm 2$ & $90 \pm 6$ & $248 \pm 22$ & $1 \pm 0.3$ \\
\hline \multicolumn{5}{|c|}{ Lines with low $S r c-418$ levels } \\
\hline LCT & $2 \pm 0.2$ & $90 \pm 8$ & $222 \pm 31$ & $0.1 \pm 0.1$ \\
\hline SHP-77 & $5 \pm 1$ & $2 \pm 1$ & $12 \pm 2$ & $0.1 \pm 0.1$ \\
\hline BHE & $1 \pm 0.1$ & $1 \pm 0.2$ & $8 \pm 2$ & $0.1 \pm 0.1$ \\
\hline \multicolumn{5}{|c|}{ Lines with low $S r c-418$ and high GJIC } \\
\hline QUDB & $7 \pm 1$ & $9 \pm 2$ & $20 \pm 4$ & $6.3 \pm 1$ \\
\hline SK-LuCi6 & $10 \pm 1$ & $8 \pm 2$ & $21 \pm 4$ & $6.5 \pm 1$ \\
\hline Rat F111 & $0.2 \pm 0.1$ & $0.2 \pm 0.1$ & $28 \pm 3$ & $5.1 \pm 1$ \\
\hline T51B & $5 \pm 1$ & $5 \pm 3$ & $18 \pm 2$ & $6.0 \pm 1$ \\
\hline E10 & $6 \pm 1$ & $6 \pm 4$ & $26 \pm 9$ & $6.0 \pm 1$ \\
\hline
\end{tabular}

${ }^{\alpha}$ Stat3-705 and Src418 levels were measured by Western blotting. Numbers represent relative values obtained by quantitation analysis, with the average of the values for Src-transduced, SK-LuCi6-Src cells taken as $100 \%$. Averages of at least three experiments \pm SEM are shown. For Stat3, data from cells grown to $50 \%$ confluence or 3 days after confluence are presented, with the average of the values for Src-transduced, SK-LuCi6-Src cells grown to $50 \%$ confluence taken as $100 \%$. The transcriptional activity values obtained paralleled the Stat3-705 phosphorylation levels indicated [23]. Rat F111: Fisher Rat fibroblasts [48]; T51B: Rat liver epithelial line [36]; E10: mouse lung epithelial type II line [37]; ${ }^{\beta}$ GJIC was assessed at 3 days after confluence.

\subsection{Effect of Src and Stat3 upon GJIC in NSCLC Lines}

An inverse relationship between Src and GJIC was noted in certain NSCLC lines [33]; A549, SK-Lu1, Calu-1, Calu-6, SW-900, SW-1573, WT-E, BEN, H1299, FR-E or SK-MES cells with high Src418 had low or undetectable gap junctional permeability, while two lines with very low Src levels (QU-DB, SK-LuCi6) had extensive GJIC (Figure 2 and Table 1). In addition, primary cells explanted and cultured from three lung cancer specimens had elevated Src418 and low GJIC [33]. These data are 
in line with the established role of $S r c$ as a GJIC suppressor. However, lines LC-T, BH-E and SHP-77 with low Src418 were found to have very low GJIC as well (Table 1), indicating that other, Src-independent factors may play a role in reducing gap junctional permeability in these cells.

Figure 2. (A) Stat3 downregulation eliminates gap junctional permeability in human lung carcinoma QU-DB cells. QU-DB cells were plated in electroporation chambers and subjected to a pulse in the presence of Lucifer yellow, following treatment with the DMSO carrier alone (a-c), or CPA7 (d-f). After washing away the unincorporated dye, cells from the same field were photographed under fluorescence $(\mathbf{b}, \mathbf{e})$ or phase contrast $(\mathbf{a}, \mathbf{d})$ illumination. In (b), cells at the edge of the conductive area, which were loaded with LY through electroporation were marked with a star, and cells at the non-electroporated area which received LY through gap junctions were marked with a dot. Arrows point to the edge of the electroporated area. (c, f): Overlay of phase-contrast and fluorescence. Magnification: $240 \times$. Note the extensive gap junctional communication in b; (B) Stat3 downregulation does not increase gap junctional permeability in human lung carcinoma A549 cells. Same as above, A549 cells. Note the absence of GJIC, even after Stat3 downregulation (e). From reference [33], reproduced with permission. Bar, $50 \mu \mathrm{m}$; (C) Stat3 downregulation reduces Cx43 levels. QU-DB cells, infected with a lentiviral vector carrying a Stat3-specific shRNA (lane 2) or not infected (lane 1), were grown to 2 days post-confluence and lysates probed for $\mathrm{Cx} 43$ or tubulin as a loading control (adapted with permission from references [33] and [38]).

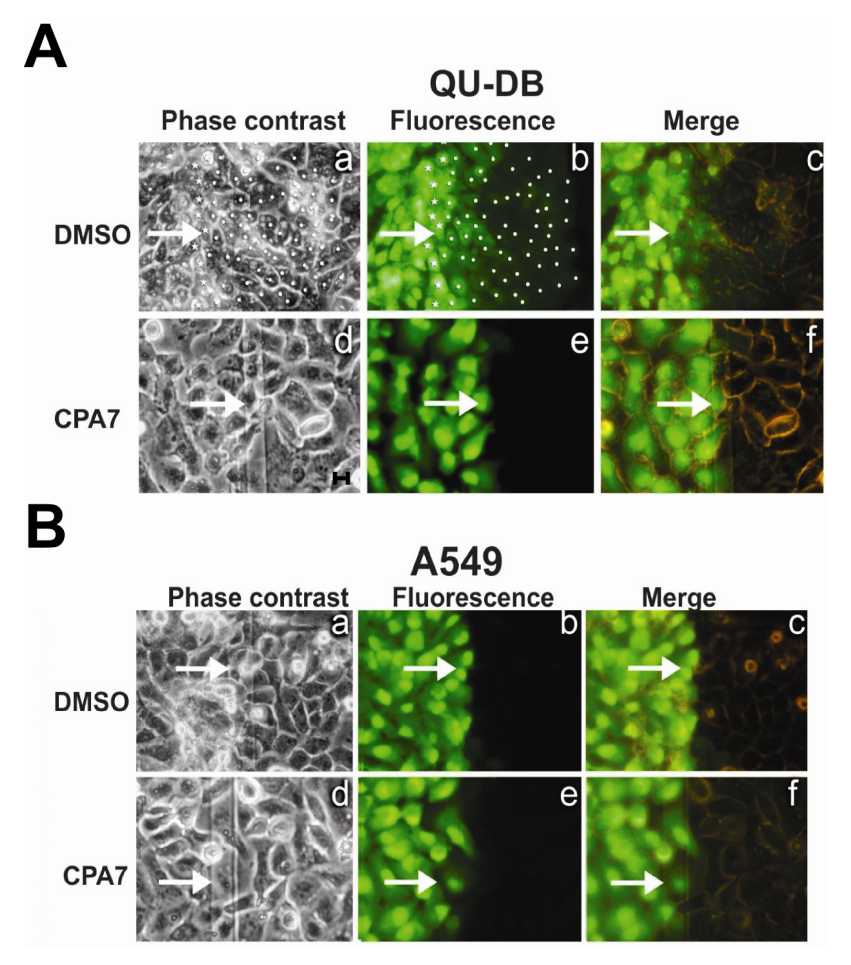


Figure 2. Cont.

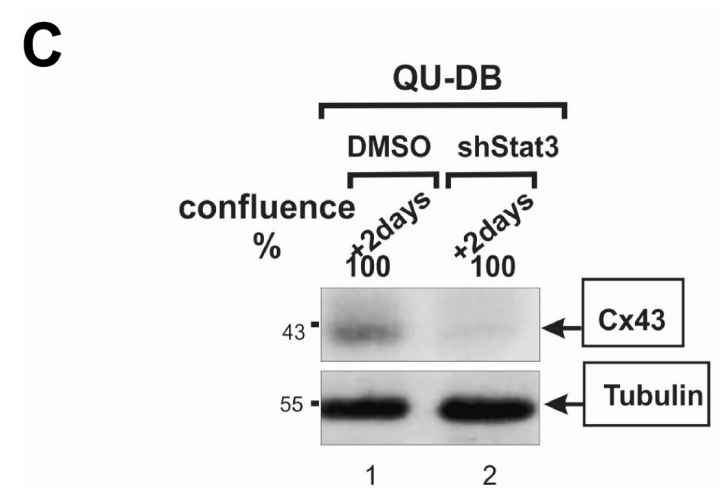

Examination of the role of Stat3 through the use of the CPA7 [40] or S3I-201 [49] inhibitors in the NSCLC lines with high Src418 showed that this treatment did not increase GJIC (e.g., line A549, Figure 2B) [33,38], in agreement with observations from T51B-Src cells [36], indicating that Stat3 is not part of a Src pathway to GJIC suppression. In addition, Stat3 inhibition with CPA7 or S3I-201 in LC-T cells which display high Stat3-705 despite the fact that Src418 levels are low, did not increase GJIC, indicating that Stat3, which is likely activated by a $S r c$-independent mechanism, is not part of a pathway leading to gap junction closure in these cells. Finally, Stat3 inhibition in BH-E and SHP-77 cells that have very low GJIC although both Src and Stat3 phosphorylation levels are low did not reinstate junctional permeability, pointing to a mechanism of gap junction closure which may be independent from the $\mathrm{Src} / \mathrm{Stat} 3$ axis altogether [38].

Examination of the effect of Stat3 inhibition upon GJIC levels in QUDB and SK-LuCi6 cells which have extensive communication indicated that Stat3 downregulation essentially abolished GJC (Figure 2A) and $\mathrm{Cx} 43$ (Figure 2C), in agreement with results from T51B cells. Therefore, rather than increasing GJIC, Stat3 inhibition eliminates gap junctional permeability, that is Stat3 activity is actually required for gap junction function in established rodent lines, as well as in certain NSCLC lines which display extensive GJIC.

\section{Discussion}

Extensive data from a number of labs demonstrated that oncogenes such as mT, $\operatorname{Src}$ or Ras can suppress GJIC [12,50]. Moreover, the results revealed that lower levels of these proteins were sufficient to eliminate gap junction function than the levels necessary for full transformation [9,51], indicating that a decrease in GJC may be an early event in neoplastic conversion. Therefore, the question arises on the role of $S r c$, an oncogene often activated in cancer, and its effector Stat3 upon GJIC.

Examination of the relationship between $\operatorname{Src}$ and Stat 3 must take cell density into account, since confluence of cultured cells itself causes a dramatic increase in Stat3, ptyr-705 phosphorylation and transcriptional activity (reviewed in $[25,26]$ ). However, despite the fact that Src is a potent Stat3 activator, $S r c$ is not involved in the density-dependent Stat3 upregulation. Therefore, at any given time-point Stat3-705 levels are the sum of the activation due to density alone (E10, T51B, QU-DB, SK-LuCi6), or density plus the Stat3 activation triggered by Src and/or other kinases (E10-Src, T51B-Src, SK-LuCi6-Src, LC-T, Calu-1, Calu-6, SW-900, SK-Lu1, A549, FR-E, WT-E) (Figure 3). Interestingly, activation of Stat 3 through cadherin engagement was found to occur despite the presence of Src, which 
is known to induce cadherin degradation [52]; apparently, the residual cadherin present is able to activate Stat3 [53].

\subsection{Stat3 Does Not Transmit Src Signals to Gap Junction Closure}

Several signal transducers besides Stat 3 are known to be downstream effectors of the Src kinase such as Ras/Raf/Erk, PI3k/Akt, the Crk-associated substrate (Cas) and others [54]. Constitutively active Ras is neoplastically transforming and can suppress GJIC [12,51]. Examination of the mechanism of Src-mediated GJIC suppression indicated that inhibition of Ras in Src-transformed, rat fibroblasts reinstated gap junctional communication [17]. Conversely, mT expression in Ras-deficient cells did not suppress GJIC [55]. Taken together, these data underline the importance of the Ras pathway in GJIC reduction by activated Src. It was also shown later that Cas is required for the $S r c$-induced, reduction in gap junctional communication [56]. In sharp contrast, Stat 3 inhibition did not restore GJIC in any of the lines examined, indicating that a role of Stat 3 in the Src-induced, GJIC suppression in these cells is unlikely, despite the fact that constitutively active Stat 3 can act as an oncogene and transform established lines [19].

Figure 3. Schematic of Stat3 activity levels as a function of density in SK-LuCi6 (A) vs. SK-LuCi6-Src (B) cells. In SK-LuCi6 cells the cadherin-triggered, Stat3 activation is resistant to $S r c$ inhibition. In transformed SK-LuCi6-Src cells however, there are two pathways of Stat3 activation: vSrc-dependent, same at all densities, and the cadherin-mediated, which is independent of Src and increases dramatically with confluence. The cadherin-mediated increase is not as pronounced however, due to the adverse effect of $\operatorname{Src}$ upon cadherins [50].

A

SK-LuCi6

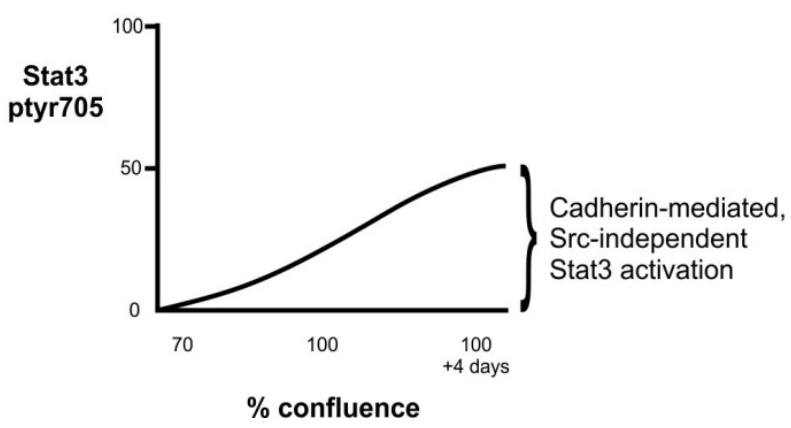

B

\section{SK-LuCi6-Src}

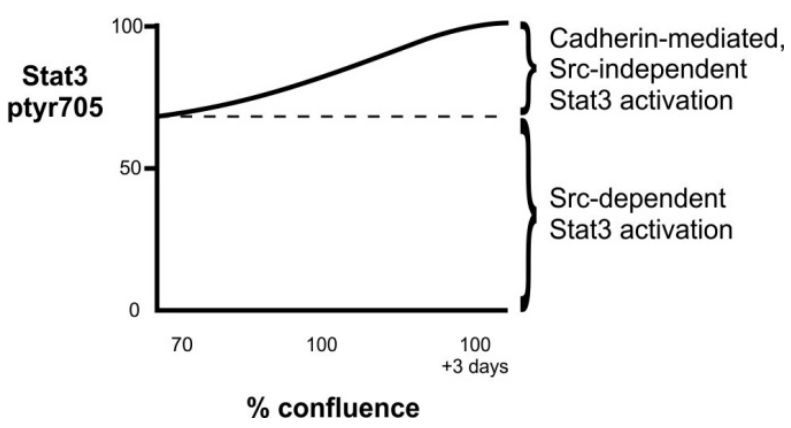

\subsection{Stat3 Plays a Positive Role in Gap Junctional Communication}

The fact that cell density upregulates Stat 3 concomitant with an increase in both Cx43 and GJIC prompted us to explore a potential positive role of Stat3 upon GJIC. Interestingly, Stat3 inhibition in non-neoplastic rodent fibroblasts and epithelial cells, as well as two NSCLC lines which exhibit extensive junctional communication (QU-DB, SK-LuCi6) abolished GJIC, indicating that Stat3 is required for the maintenance of gap junction function.

Examination of the mechanism of GJIC suppression in NSCLC revealed an inverse relationship between Src, tyr-418 phosphorylation levels and GJIC in a number of lines. Since Src is known to 
suppress gap junctional communication it is tempting to speculate that $\operatorname{Src}$ may be responsible, at least in part, for gap junction closure in these lines. Therefore, since Stat 3 is a positive regulator of GJIC and Cx43 levels, it appears that $\mathrm{Src}$ has a dual role upon GJIC; as an inhibitor, through $\mathrm{Cx} 43$ phosphorylation, but also as a GJIC activator, through Stat3 stimulation. However, the former prevails, with gap junction closure as a result.

Stat 3 downregulation caused apoptosis in all lines examined, which was more pronounced at 3 days post-confluence [33,57], the time of GJIC analysis. This hints at a link between GJIC reduction and apoptosis induced by Stat3 inhibition. In fact, results from a number of labs demonstrated that Stat3-705 activates a number of anti-apoptotic genes, such as BcL-xL, Mcl-1, survivin and Akt1 [21]. In addition, Stat3 can also inhibit apoptosis by downregulating mRNA's of mitochondrial genes, thereby reducing oxidative phosphorylation and ROS (reactive oxygen species) production [58], while ser727-phosphorylated Stat3 enhances the activity of ETC (electrotransfer chain) complexes and glycolysis, and opposes the mitochondrial permeability transition pore, thereby inhibiting apoptosis further [59-61]. Global induction of apoptosis with etoposide, cycloheximide or puromycin was shown to lead to a loss of cell coupling, probably due to caspase-3-mediated degradation of $\mathrm{Cx} 43$, in primary bovine lens epithelial and mouse NIH3T3 fibroblasts [62]. In fact, Stat3 inhibition in cells transformed by $\operatorname{Src}$ or the Large Tumor antigen of Simian Virus 40 led to apoptosis [24,33,63], possibly due to activation of the transcription factor E2F family, potent apoptosis inducers, by these oncogenes. Therefore, apoptosis induced by Stat3 downregulation in cells with high $\mathrm{Src} / \mathrm{E} 2 \mathrm{~F}$ activity may have accentuated gap junction closure. In lines such as LC-T which has low Src418, other oncogenes that activate Stat3, may also be activating the E2F family and induce apoptosis, with gap junction closure as a result. The fact that another major survival pathway, the PI3k/Akt also has a positive effect upon GJIC [64] tends to favor this interpretation. However, since Stat3 binds to and activates the Cx43 promoter, it is possible that Stat3 inhibition may downregulate $\mathrm{Cx} 43$ through a direct effect upon its mRNA as well [65-67].

In non-transformed cells with low E2F levels, grown to low densities, Stat3 inhibition was previously shown to cause merely a growth retardation. However, at high densities, such as needed for optimal gap junction formation, Stat3 inhibition leads to apoptosis [57]. This could be due to the high levels of LATS (large tumor suppressor) kinase activity which is induced by the mechanical constraints at high confluence. LATS was shown to downregulate the transcription factors YAP/TAZ (Yes associated protein/transcriptional coactivator with PDE binding motif), which are potent survival signals [26]. Therefore, apoptosis induction through a reduction in Stat3 levels or activity could explain the dramatic reduction in Cx43 and GJIC upon Stat3 pharmacological or genetic inhibition, in lines with low $\mathrm{Src} / \mathrm{E} 2 \mathrm{~F}$.

\section{Conclusions}

Although Stat3 is generally growth promoting, and in an activated form it can act as an oncogene, it does not transmit gap junction suppressing signals in any of the NSCLC cell lines examined. This holds true for cells where $S r c$ may have been responsible at least in part for GJIC suppression like the majority of NSCLC lines, but also for lines like LC-T where Src levels were found to be low, that is other oncogenes must be responsible for gap junction closure. In the contrary, Stat 3 is required for gap 
junctional communication and the maintenance of $\mathrm{Cx} 43$ levels, both in normal epithelial cells and in certain tumor lines that retain GJIC. In cells such as BH-E the gap junction closure may be independent from the $\mathrm{Src} / \mathrm{Stat} 3$ axis altogether.

The role of connexins in cancer is complex. Numerous models support the idea that connexins are tumor suppressors at the early stages of carcinogenesis. However, recent evidence indicated a positive role for connexins in facilitating metastasis ([68-70], reviewed in [71]). Therefore, inhibition of the Stat3/Cx axis might be beneficial at late stages of cancer progression. This novel role of Stat 3 in gap junction function may be an important regulatory step in the progression of tumors that exploit such a pathway.

\section{Acknowledgments}

We would like to thank Mike Baird and Shallyn Littlefield for CPA7 synthesis, David Kaplan of the University of Toronto for the Adenovirus vectors and Kevin Firth, of Ask Sciences products, Kingston, Ontario for engineering design. The financial assistance of the Canadian Institutes of Health Research (CIHR), the Canadian Breast Cancer Foundation (Ontario Chapter), the Natural Sciences and Engineering Research Council of Canada (NSERC), the Canadian Breast Cancer Research Alliance, the Ontario Centers of Excellence, the Breast Cancer Action Kingston and the Clare Nelson bequest fund through grants to LR is gratefully acknowledged. SG was supported by an NSERC studentship and a Queen's University Graduate Award. RA was supported by a CIHR studentship and a Doctoral Women's Health Scholars Award from the Ontario Council on Graduate Studies. MG was supported by a postdoctoral fellowship from the US Army Breast Cancer Program, the Ministry of Research and Innovation of the Province of Ontario and the Advisory Research Committee of Queen's University.

\section{Author Contributions}

Stephanie Guy together with Mulu Geletu did the bulk of the benchwork. Rozanne Arulanandam conducted some of the experiments, especially on GJIC in the lung cancer lines. Leda Raptis conceived of the study, drafted the paper and supervised throughout.

\section{Conflicts of Interest}

The authors declare no conflicts of interest.

\section{References}

1. Sohl, G.; Willecke, K. An update on connexin genes and their nomenclature in mouse and man. Cell Commun. Adhes. 2003, 10, 173-180.

2. Laird, D.W. The gap junction proteome and its relationship to disease. Trends Cell. Biol. 2010, 20, 92-101.

3. Laird, D.W. Life cycle of connexins in health and disease. Biochem. J. 2006, 394, 527-543.

4. Sohl, G.; Willecke, K. Gap junctions and the connexin protein family. Cardiovasc. Res. 2004, 62, $228-232$. 
5. Beardslee, M.A.; Laing, J.G.; Beyer, E.C.; Saffitz, J.E. Rapid turnover of connexin43 in the adult rat heart. Circ. Res. 1998, 83, 629-635.

6. Vanslyke, J.K.; Naus, C.C.; Musil, L.S. Conformational maturation and post-ER multisubunit assembly of gap junction proteins. Mol. Biol. Cell 2009, 20, 2451-2463.

7. Gaietta, G.; Deerinck, T.J.; Adams, S.R.; Bouwer, J.; Tour, O.; Laird, D.W.; Sosinsky, G.E.; Tsien, R.Y.; Ellisman, M.H. Multicolor and electron microscopic imaging of connexin trafficking. Science 2002, 296, 503-507.

8. Lin, R.; Warn-Cramer, B.J.; Kurata, W.E.; Lau, A.F. v-Src Phosphorylation of connexin 43 on Tyr247 and Tyr265 disrupts gap junctional communication. J. Cell Biol. 2001, 154, 815-827.

9. Raptis, L.; Brownell, H.L.; Firth, K.L.; MacKenzie, L.W. A novel technique for the study of intercellular, junctional communication; electroporation of adherent cells on a partly conductive slide. DNA Cell Biol. 1994, 13, 963-975.

10. Schaffhausen, B.S.; Roberts, T.M. Lessons from polyoma middle T antigen on signaling and transformation: A DNA tumor virus contribution to the war on cancer. Virology 2009, 384, 304-316.

11. Grammatikakis, N.; Vultur, A.; Ramana, C.V.; Siganou, A.; Schweinfest, C.W.; Raptis, L. The role of Hsp90N, a new member of the Hsp90 family, in signal transduction and neoplastic transformation. J. Biol. Chem. 2002, 277, 8312-8320.

12. Brownell, H.L.; Narsimhan, R.; Corbley, M.J.; Mann, V.M.; Whitfield, J.F.; Raptis, L. Ras is involved in gap junction closure in mouse fibroblasts or preadipocytes but not in differentiated adipocytes. DNA Cell Biol. 1996, 15, 443-451.

13. Atkinson, M.M.; Sheridan, J.D. Altered junctional permeability between cells transformed by v-ras, v-mos, or v-src. Am. J. Physiol. 1988, 255, C674-C683.

14. Solan, J.L.; Lampe, P.D. Connexin43 phosphorylation: Structural changes and biological effects. Biochem. J. 2009, 419, 261-272.

15. Geletu, M.; Trotman-Grant, A.; Raptis, L. Mind the gap; regulation of gap junctional, intercellular communication by the SRC oncogene product and its effectors. Anticancer Res. 2012, 32, 4245-4250.

16. Pahujaa, M.; Anikin, M.; Goldberg, G.S. Phosphorylation of connexin43 induced by Src: Regulation of gap junctional communication between transformed cells. Exp. Cell Res. 2007, 313, 4083-4090.

17. Ito, S.; Ito, Y.; Senga, T.; Hattori, S.; Matsuo, S.; Hamaguchi, M. v-Src requires Ras signaling for the suppression of gap junctional intercellular communication. Oncogene 2006, 25, 2420-2424.

18. Zhang, Y.; Turkson, J.; Carter-Su, C.; Smithgall, T.; Levitzki, A.; Kraker, A.; Krolewski, J.J.; Medveczky, P.; Jove, R. Activation of Stat3 in v-Src transformed fibroblasts requires cooperation of Jak1 kinase activity. J. Biol. Chem. 2000, 275, 24935-24944.

19. Bromberg, J.F.; Wrzeszczynska, M.H.; Devgan, G.; Zhao, Y.; Pestell, R.G.; Albanese, C.; Darnell, J.E., Jr. Stat3 as an oncogene. Cell 1999, 98, 295-303.

20. Hung, W.; Elliott, B. Co-operative effect of c-Src tyrosine kinase and Stat3 in activation of hepatocyte growth factor expression in mammary carcinoma cells. J. Biol. Chem. 2001, 276, 12395-12403.

21. Yu, H.; Pardoll, D.; Jove, R. STATs in cancer inflammation and immunity: A leading role for STAT3. Nat. Rev. Cancer 2009, 9, 798-809.

22. Arulanandam, R.; Vultur, A.; Cao, J.; Carefoot, E.; Truesdell, P.; Elliott, B.; Larue, L.; Feracci, H.; Raptis, L. Cadherin-cadherin engagement promotes survival via Rac/Cdc42 and Stat3. Mol. Cancer Res. 2009, 17, 1310-1327. 
23. Vultur, A.; Cao, J.; Arulanandam, R.; Turkson, J.; Jove, R.; Greer, P.; Craig, A.; Elliott, B.E.; Raptis, L. Cell to cell adhesion modulates Stat3 activity in normal and breast carcinoma cells. Oncogene 2004, 23, 2600-2616.

24. Vultur, A.; Arulanandam, R.; Turkson, J.; Niu, G.; Jove, R.; Raptis, L. Stat3 is required for full neoplastic transformation by the Simian Virus 40 large tumor antigen. Mol. Biol. Cell 2005, 16, 3832-3846.

25. Raptis, L.; Arulanandam, R.; Vultur, A.; Geletu, M.; Chevalier, S.; Feracci, H. Beyond structure, to survival: Stat3 Activation by cadherin engagement. Biochem. Cell Biol. 2009, 87, 835-843.

26. Geletu, M.; Guy, S.; Arulanandam, R.; Feracci, H.; Raptis, L. Engaged for survival: From cadherin ligation to Stat3 activation. JAK-STAT 2013, 2, e27363.

27. El-Fouly, M.H.; Trosko, J.E.; Chang, C.C. Scrape-loading and dye transfer: A rapid and simple technique to study gap junctional intercellular communication. Exp. Cell Res. 1987, 168, 442-430.

28. Goldberg, G.S.; Bechberger, J.F.; Naus, C.C. A pre-loading method of evaluating gap junctional communication by fluorescent dye transfer. Biotechniques 1995, 18, 490-497.

29. Wade, M.H.; Trosko, J.E.; Schindler, M. A fluorescence photobleaching assay of gap junction-mediated communication between human cells. Science 1986, 232, 525-528.

30. Raptis, L.; Vultur, A.; Brownell, H.L.; Tomai, E.; Anagnostopoulou, A.; Arulanandam, R.; Cao, J.; Firth, K.L. Electroporation Protocols; Li, S., Ed.; The Humana Press Inc.: Totowa, NJ, USA, 2008; pp. 167-183.

31. Anagnostopoulou, A.; Cao, J.; Vultur, A.; Firth, K.L.; Raptis, L. Examination of gap junctional, intercellular communication by in situ electroporation on two co-planar indium-tin oxide electrodes. Mol. Oncol. 2007, 1, 226-231.

32. Tomai, E.; Brownell, H.L.; Tufescu, T.; Reid, K.; Raptis, S.; Campling, B.G.; Raptis, L. A functional assay for intercellular, junctional communication in cultured human lung carcinoma cells. Lab. Investig. 1998, 78, 639-640.

33. Geletu, M.; Arulanandam, R.; Greer, S.; Trotman-Grant, A.; Tomai, E.; Raptis, L. Stat3 is a positive regulator of gap junctional intercellular communication in cultured, human lung carcinoma cells. BMC Cancer 2012, 12, doi:10.1186/1471-2407-12-605.

34. Brownell, H.L.; Lydon, N.; Schaefer, E.; Roberts, T.M.; Raptis, L. Inhibition of Epidermal Growth Factor-mediated ERK1/2 activation by in situ electroporation of nonpermeant [(alkylamino)methyl]acrylophenone derivatives. DNA Cell Biol. 1998, 17, 265-274.

35. Tomai, E.; Brownell, H.L.; Tufescu, T.; Reid, K.; Raptis, L. Gap junctional communication in lung carcinoma cells. Lung Cancer 1999, 23, 223-231.

36. Geletu, M.; Chaize, C.; Arulanandam, R.; Vultur, A.; Kowolik, C.; Anagnostopoulou, A.; Jove, R.; Raptis, L. Stat3 activity is required for gap junctional permeability in normal epithelial cells and fibroblasts. DNA Cell Biol. 2009, 28, 319-327.

37. Vultur, A.; Tomai, E.; Peebles, K.; Malkinson, A.M.; Grammatikakis, N.; Forkert, P.G.; Raptis, L. Gap junctional, intercellular communication in cells from urethane-induced tumors in $\mathrm{A} / \mathrm{J}$ mice. DNA Cell Biol. 2003, 22, 33-40.

38. Geletu, M.; Guy, S.; Raptis, L. Effects of SRC and STAT3 upon gap junctional, intercellular communication in lung cancer lines. Anticancer Res. 2013, 33, 4401-4410. 
39. Wei, C.J.; Francis, R.; Xu, X.; Lo, C.W. Connexin43 associated with an N-cadherin-containing multiprotein complex is required for gap junction formation in NIH3T3 cells. J. Biol. Chem. 2005, 280, 19925-19936.

40. Turkson, J.; Zhang, S.; Palmer, J.; Kay, H.; Stanko, J.; Mora, L.B.; Sebti, S.; Yu, H.; Jove, R. Inhibition of constitutive signal transducer and activator of transcription 3 activation by novel platinum complexes with potent antitumor activity. Mol. Cancer Ther. 2004, 3, 1533-1542.

41. Littlefield, S.L.; Baird, M.C.; Anagnostopoulou, A.; Raptis, L. Synthesis, characterization and Stat3 inhibitory properties of the prototypical platinum(IV) anticancer drug, $\left[\mathrm{PtCl}_{3}\left(\mathrm{NO}_{2}\right)\left(\mathrm{NH}_{3}\right)_{2}\right]$ (CPA-7). Inorg. Chem. 2008, 47, 2798-2804.

42. Siddiquee, K.; Zhang, S.; Guida, W.C.; Blaskovich, M.A.; Greedy, B.; Lawrence, H.R.; Yip, M.L.; Jove, R.; McLaughlin, M.M.; Lawrence, N.J.; et al. Selective chemical probe inhibitor of Stat3, identified through structure-based virtual screening, induces antitumor activity. Proc. Natl. Acad. Sci. USA 2007, 104, 7391-7396.

43. Trotman-Grant, A.; Geletu, M.; Raptis, L. Constitutively active, Signal transducer and activator of transcription-3; an oncogene that increases gap junctional communication. Queen's University, Kingston, ON, Canada. Unpublished work, 2014.

44. Masaki, T.; Igarashi, K.; Tokuda, M.; Yukimasa, S.; Han, F.; Jin, Y.J.; Li, J.Q.; Yoneyama, H.; Uchida, N.; Fujita, J.; et al. pp60c-src Activation in lung adenocarcinoma. Eur. J. Cancer 2003, $39,1447-1455$.

45. Zhang, J.; Kalyankrishna, S.; Wislez, M.; Thilaganathan, N.; Saigal, B.; Wei, W.; Ma, L.; Wistuba, I.I.; Johnson, F.M.; Kurie, J.M. SRC-family kinases are activated in non-small cell lung cancer and promote the survival of epidermal growth factor receptor-dependent cell lines. Am. J. Pathol. 2007, 170, 366-376.

46. Song, L.; Turkson, J.; Karras, J.G.; Jove, R.; Haura, E.B. Activation of Stat3 by receptor tyrosine kinases and cytokines regulates survival in human non-small cell carcinoma cells. Oncogene 2003, 22, 4150-4165.

47. Byers, L.A.; Sen, B.; Saigal, B.; Diao, L.; Wang, J.; Nanjundan, M.; Cascone, T.; Mills, G.B.; Heymach, J.V.; Johnson, F.M. Reciprocal regulation of c-Src and STAT3 in non-small cell lung cancer. Clin. Cancer Res. 2009, 15, 6852-6861.

48. Raptis, L.; Lamfrom, H.; Benjamin, T.L. Regulation of cellular phenotype and expression of polyomavirus middle T antigen in rat fibroblasts. Mol. Cell. Biol. 1985, 5, 2476-2486.

49. Turkson, J.; Zhang, S.; Mora, L.B.; Burns, A.; Sebti, S.; Jove, R. A novel platinum compound that inhibits constitutive Stat 3 signaling and induces cell cycle arrest and apoptosis of malignant cells. J. Biol. Chem. 2005, 280, 32979-32988.

50. Azarnia, R.; Loewenstein, W.R. Polyomavirus middle T antigen downregulates junctional cell-to-cell communication. Mol. Cell. Biol. 1987, 7, 946-950.

51. Brownell, H.L.; Whitfield, J.F.; Raptis, L. Elimination of intercellular junctional communication requires lower Ras ${ }^{\text {leu61 }}$ levels than stimulation of anchorage-independent proliferation. Cancer Detect. Prev. 1997, 21, 289-294.

52. Wadhawan, A.; Smith, C.; Nicholson, R.I.; Barrett-Lee, P.; Hiscox, S. Src-mediated regulation of homotypic cell adhesion: Implications for cancer progression and opportunities for therapeutic intervention. Cancer Treat. Rev. 2011, 37, 234-241. 
53. Guy, S.; Geletu, M.; Arulanandam, R.; Raptis, L. Cadherin-11 function is required for full neoplastic transfomation by vSrc. Queen's University, Kingston, ON, Canada. Unpublished work, 2014.

54. Aleshin, A.; Finn, R.S. SRC: A century of science brought to the clinic. Neoplasia 2010, 12, 599-607.

55. Brownell, H.L.; Whitfield, J.F.; Raptis, L. Cellular Ras partly mediates gap junction closure by the polyoma virus middle Tumor antigen. Cancer Lett. 1996, 103, 99-106.

56. Shen, Y.; Khusial, P.R.; Li, X.; Ichikawa, H.; Moreno, A.P.; Goldberg, G.S. SRC utilizes Cas to block gap junctional communication mediated by connexin43. J. Biol. Chem. 2007, 282, 18914-18921.

57. Anagnostopoulou, A.; Vultur, A.; Arulanandam, R.; Cao, J.; Turkson, J.; Jove, R.; Kim, J.S.; Glenn, M.; Hamilton, A.D.; Raptis, L. Differential effects of Stat3 inhibition in sparse vs. confluent normal and breast cancer cells. Cancer Lett. 2006, 242, 120-132.

58. Gao, X.; Wang, H.; Yang, J.J.; Liu, X.; Liu, Z.R. Pyruvate kinase M2 regulates gene transcription by acting as a protein kinase. Mol. Cell 2012, 45, 598-609.

59. Wegrzyn, J.; Potla, R.; Chwae, Y.J.; Sepuri, N.B.; Zhang, Q.; Koeck, T.; Derecka, M.; Szczepanek, K.; Szelag, M.; Gornicka, A.; et al. Function of mitochondrial Stat3 in cellular respiration. Science 2009, 323, 793-797.

60. Gough, D.J.; Corlett, A.; Schlessinger, K.; Wegrzyn, J.; Larner, A.C.; Levy, D.E. Mitochondrial STAT3 supports Ras-dependent oncogenic transformation. Science 2009, 324, 1713-1716.

61. Demaria, M.; Poli, V. From the nucleus to the mitochondria and back: The odyssey of a multitask STAT3. Cell Cycle 2011, 10, 3221-3222.

62. Theiss, C.; Mazur, A.; Meller, K.; Mannherz, H.G. Changes in gap junction organization and decreased coupling during induced apoptosis in lens epithelial and NIH-3T3 cells. Exp. Cell Res. 2007, 313, 38-52.

63. Anagnostopoulou, A.; Vultur, A.; Arulanandam, R.; Cao, J.; Turkson, J.; Jove, R.; Kim, J.S.; Glenn, M.; Hamilton, A.D.; Raptis, L. Role of Stat3 in normal and SV40 transformed cells. Res. Trends 2006, 2, 93-103.

64. Geletu, M.; Guy, S.; Greer, S.; Raptis, L. Differential effects of polyoma virus middle tumor antigen mutants upon gap junctional, intercellular communication. Queen's University, Kingston, ON, Canada. Unpublished work, 2014.

65. Ozog, M.A.; Bernier, S.M.; Bates, D.C.; Chatterjee, B.; Lo, C.W.; Naus, C.C. The complex of ciliary neurotrophic factor-ciliary neurotrophic factor receptor alpha up-regulates connexin 43 and intercellular coupling in astrocytes via the Janus tyrosine kinase/signal transducer and activator of transcription pathway. Mol. Biol. Cell 2004, 15, 4761-4774.

66. Rajasingh, J.; Bord, E.; Hamada, H.; Lambers, E.; Qin, G.; Losordo, D.W.; Kishore, R. STAT3-dependent mouse embryonic stem cell differentiation into cardiomyocytes: Analysis of molecular signaling and therapeutic efficacy of cardiomyocyte precommitted $\mathrm{mES}$ transplantation in a mouse model of myocardial infarction. Circ. Res. 2007, 101, 910-918.

67. Andersson, H.; Brittebo, E. Proangiogenic effects of environmentally relevant levels of bisphenol A in human primary endothelial cells. Arch. Toxicol. 2012, 86, 465-474.

68. Li, Q.; Omori, Y.; Nishikawa, Y.; Yoshioka, T.; Yamamoto, Y.; Enomoto, K. Cytoplasmic accumulation of connexin32 protein enhances motility and metastatic ability of human hepatoma cells in vitro and in vivo. Int. J. Cancer 2007, 121, 536-546. 
69. Ezumi, K.; Yamamoto, H.; Murata, K.; Higashiyama, M.; Damdinsuren, B.; Nakamura, Y.; Kyo, N.; Okami, J.; Ngan, C.Y.; Takemasa, I.; et al. Aberrant expression of connexin 26 is associated with lung metastasis of colorectal cancer. Clin. Cancer Res. 2008, 14, 677-684.

70. Elzarrad, M.K.; Haroon, A.; Willecke, K.; Dobrowolski, R.; Gillespie, M.N.; Al Mehdi, A.B. Connexin-43 upregulation in micrometastases and tumor vasculature and its role in tumor cell attachment to pulmonary endothelium. BMC Med. 2008, 6, e20.

71. Naus, C.C.; Laird, D.W. Implications and challenges of connexin connections to cancer. Nat. Rev. Cancer 2010, 10, 435-441.

(C) 2014 by the authors; licensee MDPI, Basel, Switzerland. This article is an open access article distributed under the terms and conditions of the Creative Commons Attribution license (http://creativecommons.org/licenses/by/3.0/). 